\title{
TOWARDS CLOUD DRIVEN SEMANTIC ANNOTATION
}

\author{
Oluwasegun Adedugbe, Elhadj Benkhelifa and Russell Campion \\ Faculty of Computing and Digital Technologies \\ Staffordshire University \\ Stoke-on-Trent, UK ST4 2DE \\ oluwasegun.adedugbe@research.staffs.ac.uk
}

\begin{abstract}
Semantic Web Technologies have been an active research area for some time and they are concerned with the development of technological concepts and artefacts that can drive the much elusive semantic web. The idea of a semantic web is a web which comprises of data with well-defined meaning. It is also a web that is context-aware in nature, whereby web documents are easily understandable and able to be processed by machines based on the underlying meaning provided for the documents by making use of annotation data (i.e. metadata). While several concepts have been proposed to drive the semantic web, none has so far demonstrated potentials to transform the current Web 2.0 to a truly semantic Web 3.0. With the advent of diverse technological innovations such as internet of things, cloud computing, big data analytics, etc. it is pertinent to review the state-of-the-art for semantic annotation and how it can be impacted by any of these technologies. This paper provides a review of semantic annotation state-of-the-art and how cloud computing as a paradigm can impact on it.
\end{abstract}

Keywords - Semantic Web, Cloud Computing, Semantic Annotation, Semantic Technologies, Annotation Data.

\section{INTRODUCTION}

The amount of data on the Web today is very enormous. The growth rate of the Web is even more sporadic with claims that up to 700 hours of video and over 700,000 Facebook posts are made in a minute, amongst several other massive sources of data addition to the Web [1]. With the growth of the Web has been significant advancements in computing technologies (both hardware and software) to deliver web content across different media such as smart phones, mobile devices, tablets, etc. alongside traditional devices such as desktops, laptops, etc.

The Web has transited from a static to a social one. While the static web (Web 1.0) was predominantly an online repository for read-only data, the social web (Web 2.0) saw the emergence of interactive web pages with database-driven, dynamic content. Also, mission-critical transactions online came with the second phase of the Web [2]. The social context emanated from the fact that both content providers and content consumers on the Web could interact and generate content for web pages. A semantic web is emerging; one in which web documents are annotated with descriptive data (i.e. metadata) in such a way that machines can understand and process these documents rather than just presenting them without any context. With the metadata annotation to web documents, they become web data and machines can then provide context; such as differentiating between 'Jaguar' as an automobile and 'Jaguar' as an animal.

While there have been several efforts both in academia and industry towards a global semantic web, its evolvement has been plagued with a lot of challenges.

These include the tedious nature of annotating web documents with metadata, limitations with tools/methods used in annotation [3], consistency between metadata and web data when either of them changes and lots more [4]. With the emergence and prominence of cloud computing as a new computing paradigm, offering several new capabilities, this paper considers how it can be used as leverage to provide and enhance solutions for some of the semantic web challenges towards the availability of effective and efficient tools/methods to drive the semantic web.

\section{SEMANTIC WEB}

The Semantic Web is a concept that defines a web in which an underlining meaning is provided for data on the Web to make it context-aware and relationships are established between different web data by linking them together based on properties that define types of relationships [5]. Data on the Web is annotated with metadata through annotation techniques and different data linked together to form a knowledge base or repository [3]. Two major areas of Semantic Web Technology come into play for this purpose; semantic annotation and ontology engineering. While semantic annotation is the metadata-to-data annotation, ontology engineering defines a wide range of activities to provide ontologies for generating metadata.

The Semantic Web provides accessibility to resources via a URI (Uniform Resource Identifier), which can be a URN (Uniform Resource Namespace) or a URL (Uniform Resource Locator). With the semantic web addressing the challenges of the existing Web (or better known as Web 2.0), it enables merging of data sets (such as the linked open graph), data 
browsing as well as targeted search and automated agents on the web, etc. [6]. Furthermore, the implementation of semantic web technologies would enhance the Web with several capabilities [3], such as the following:

- Automated Data Linkage on the web based on relationships between annotated web documents.

- Automated Data Integration based on the existence of relational links between different data. This is as opposed to sourcing for data from various sources and trying to manually put them together as a unit.

- Automated Data Service Life Cycle for the management of web data through its entire life cycle. This includes the initial phase of data, data migration, etc. until the data becomes obsolete.

- Precision and Recall. While the precision index measures the quality of the results returned from a search, the recall index measures the completeness of the results.
Despite the several semantic web capabilities, its implementation is faced with challenges. These have been categorized into execution-related, implementation-related and generic challenges, as discussed in the next section.

\section{SEMANTIC WEB CHALLENGES}

Execution-related challenges: There are issues with methods for information extraction and retrieval (IER) from web documents. While some semantic annotation tools utilize traditional IER methods such as named entity recognition, Hidden Markov's Model, etc., [4] another school of thought believes the development and use of web IER methods as opposed to traditional ones would yield better results [7]. Examples of web IER methods include TextRunner, KnowItAll, KnowItNow, etc. These creates a wide range of processes for semantic annotation based on the IER method deployed.

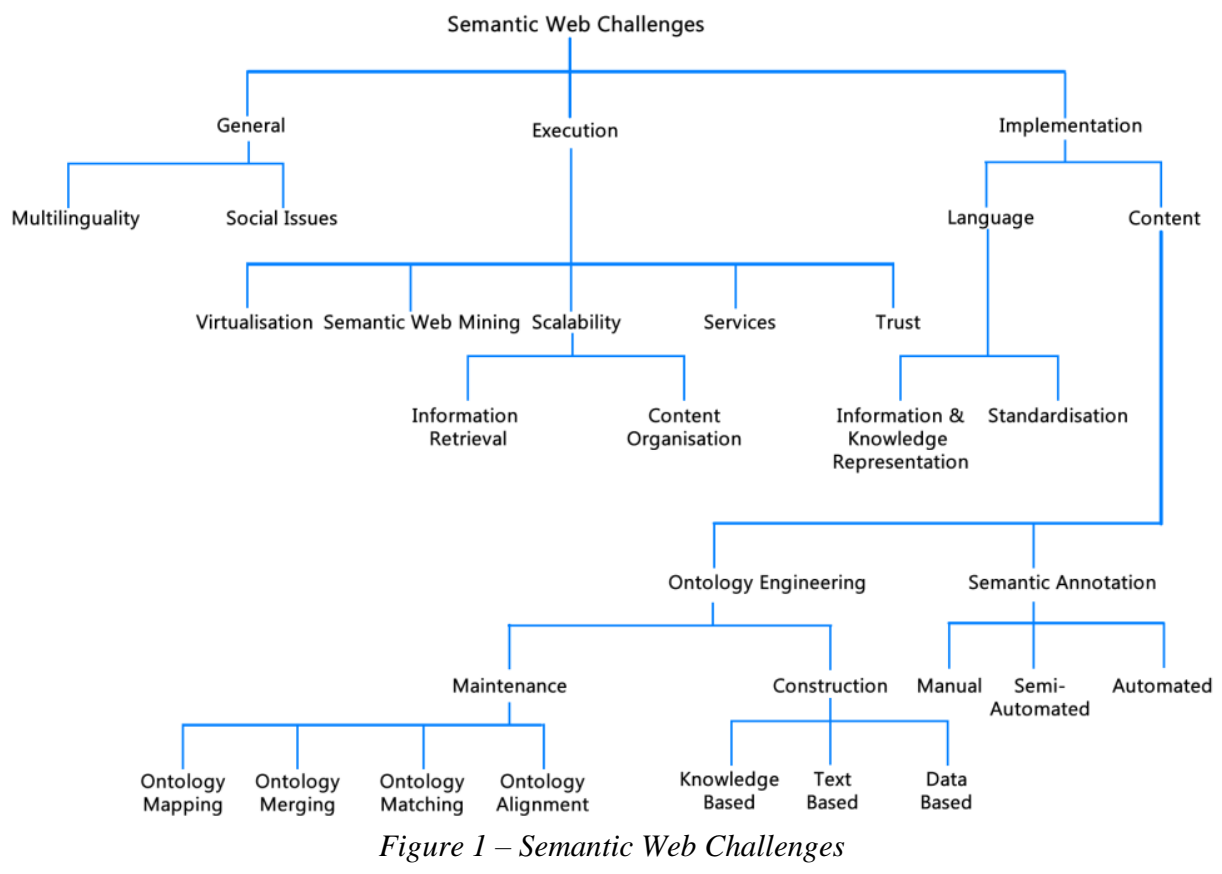

Also, there are issues with scalability, as most existing semantic annotation tools have been developed to utilize specific ontologies or run on a specific platform, thereby limiting its scope of adoption for large-scale semantic annotation. For the web to become truly semantic in nature, a semantic annotation tool that is portable in nature, easily accessible and robust enough to accept different ontologies for semantic annotation would be required [8]. Furthermore, issues relating to services and trust exist, in which there are no W3C-compliant standards usable and deployable by the public in those domains for web applications [9].

Implementation-related challenges: The major challenges in this area are focused on content for the semantic web and the means of adding them. The additional content from semantic annotation are ontology-generated metadata which are used to annotate web data for the provision of an underlying meaning and context-awareness. Based on available tools and techniques, the generation and availability of these metadata has been a daunting task due to several ontology engineering challenges such as ontology development or ontology integration. Ontology Integration is one of several other 'Ontology Engineering' challenges. Ontology Integration can be implemented in several ways such as ontology mapping, merging, alignment, elucidation, optimization, self-learning, etc. [10]. The other major area is the means of adding these additional content (i.e. metadata) when available. There are manual, semi-automatic and automatic semantic annotation methods and they all present strengths and weaknesses. 
General challenges: Some of the general semantic web challenges identified in research include multilingualism and social issues. Multilingualism refers to issues relating to the translation of ontologies and ontology-generated annotations from one language to another [11]. The task of developing ontologies for different languages is time-consuming and cumbersome, considering the need for regular updates as well. While translating ontologies from one language to another is

\section{SEMANTIC ANNOTATION}

Semantic annotation from a human perspective and from a machine perspective are slightly different. From the human perspective, an annotation could be regarded as semantic if it provides meaning or additional meaning for a piece of data to the human. This implies that tagging, labelling, mentions, linguistics annotation, corpus, etc. would be regarded as semantic annotation from a human point of view. The diagram below illustrates semantic annotation from a human point of view:

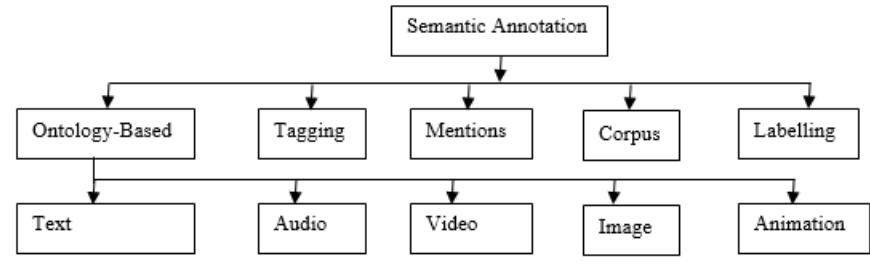

Figure 2 - Semantic Annotation from a human point of view

However, from a machine point of view, these types of annotation cannot be regarded as semantic because machines cannot understand and/or process them. For machines to understand and/or process annotation, a middle layer is required between the machine and the annotation and this middle layer can be provided by an ontology. This research is focused on an ontology-based semantic annotation, which is the requirement for the semantic web. The semantic annotation for the semantic web can be illustrated with the diagram below:

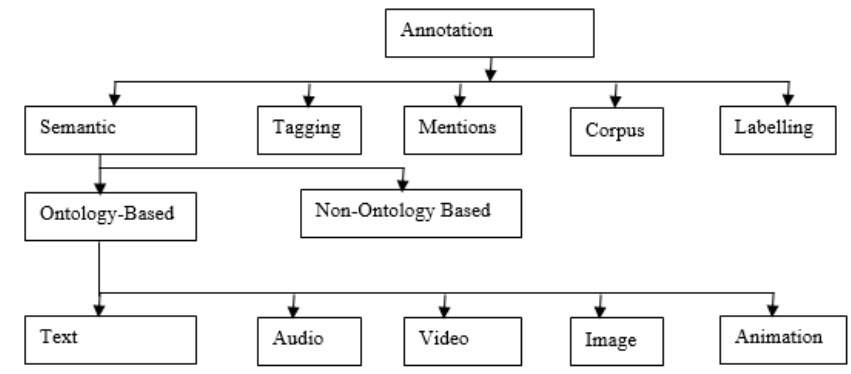

Figure 3 - Annotation from a machine point of view

There are other types of annotation which are not semantic in nature as depicted in the diagram above. This implies that such annotation does not carry a meaning understandable and process-able by machines. Often, they only provide additional information for humans. For an annotation to be semantic in nature, it needs to be driven by a machine-readable knowledge favored over developing new ones for each language, existing translation standards are not matured enough and results into inaccurate translations [12]. Also, social challenges exist such as consensus on taxonomies or data dictionaries for specific knowledge domains between experts in the field. These taxonomies or data dictionaries are required for ontology development. The diagram below represents some of these challenges and the category they fall under and the next section focuses on semantic annotation.

base such as ontologies and they constitute an abstract of the real world. They can also be referred to as a data model, since they are in an abstract form of actual data. Ontologies can be defined as an "explicit formal specification of a conceptualization" [13]

Semantic Annotation is an implementation-related challenge. In this context, the metadata; derived from an ontology or a collection of ontologies is added to web data in other to provide an underlying meaning for the web data [14]. This also implies that computers will be able to understand and process the web data due to the associated metadata. While semantic annotation can be implemented in very many ways using diverse types of tools and/or methods, the concept can be illustrated with a generic (or high-level) step-by-step process which highlights the distinct phases of the process. The major phases can be defined as (i) web document identification, (ii) information extraction and retrieval, (iii) generation of metadata and (iv) metadata-to-data annotation. There are three diverse ways of semantically annotating web data. They are manual, semi-automatic and automatic annotations.

Manual Annotation: As the name implies, this involves a manual process of adding metadata to data. This is not a feasible solution as it is a very cumbersome, tedious and timeconsuming process [15]. With the vast amount of data on the Web, this solution would not just work. Some examples of manual annotation tools include Annotator, GATE Teamware, WebAnno, Amaya, etc.

Semi-Automatic Annotation: With this type of annotation, the process is partly automated and partly manual. The weight of how much automation or manual work involved varies from one approach to another based on the different tools available [16]. For most tools, the Information Extraction stage of semantic annotation is automated while the rest of the process is manual. This approach to annotation still faces most of the challenges of manual annotation (such as being timeconsuming and tedious) and cannot be adopted as the means for annotating over 7 billion web pages available on the Web today. Some of the available semi-automatic tools include OntoMat, Melita, etc. [4].

Automatic Annotation: Manual and semi-automatic semantic annotation are very arduous, tedious, time-consuming and error prone tasks. They are not just feasible for adoption by 
developers, site administrators, content editors, etc. for use as means of annotating web pages with semantic data [17]. Automatic Annotation defines a fully automated approach to semantic annotation. In this case the annotation process is not expected to require any human intervention, instead is to be fully implemented by machines. This eradicates the issues associated with both manual and semi-automatic semantic annotation, as the process is fast and machine-processed. The downside however to automatic annotation relative to semiautomatic annotation is the fact that the automation usually results in a lesser annotation accuracy and since it is fully automated, is not checked for such errors within the process. Nevertheless, the automation process can be repeated over several loops to increase the accuracy with the use of a large data set for training the algorithm implemented for the automation process.

Also, the availability of annotation accuracy measurement indexes means the automation can be tested for accuracy. The widely accepted indexes are Precision, Recall and F-Measure [18]. Automation for semantic annotation remains the major viable means of annotating existing web data as well as the vast amount added to it on a momentary basis. While there have been several automatic annotation tools, most of them are only research-based and yet to be implemented. Several others have become obsolete, unsupported or unavailable for use. Furthermore, an all-in-one automatic semantic annotation platform that is scalable enough for the Web and captures the entire process of semantic annotation, with additional features such as ontology mapping (or engineering, as the case may be), ontology auto-update, annotation data dynamicity, reusage, sharing and auto-update is currently unavailable. SemTag, which uses a Seeker engine for automatic semantic annotation is one of the closest to the above described platform. However, it is still vastly limited in many ways, more predominantly because it's metadata source is a taxonomy (known as TAP) as compared to the possibility of openness to be used by any OWL-compliant ontology [19]. The following table presents a comparison of the three types of semantic annotation described above:

Table 1 - Comparison of semantic annotation methods

\begin{tabular}{|l|l|l|l|}
\hline & $\begin{array}{c}\text { Manual } \\
\text { Annotation }\end{array}$ & $\begin{array}{l}\text { Semi- } \\
\text { Automated } \\
\text { Annotation }\end{array}$ & $\begin{array}{l}\text { Automated } \\
\text { Annotation }\end{array}$ \\
\hline Description & $\begin{array}{l}\text { The process } \\
\text { in which } \\
\text { humans } \\
\text { manually } \\
\text { add } \\
\text { metadata to } \\
\text { data. }\end{array}$ & $\begin{array}{l}\text { The process } \\
\text { of } \\
\text { automating } \\
\text { certain tasks } \\
\text { within the } \\
\text { annotation } \\
\text { process of } \\
\text { data. One } \\
\text { major task } \\
\text { often } \\
\text { of } \\
\text { eliminating } \\
\text { the } \\
\text { requirement } \\
\text { of a human } \\
\text { intervention } \\
\text { information } \\
\text { in the } \\
\text { extraction }\end{array}$ & $\begin{array}{l}\text { annotation } \\
\text { process for } \\
\text { web data, } \\
\text { making it } \\
\text { fully }\end{array}$ \\
\hline
\end{tabular}

\begin{tabular}{|c|c|c|c|}
\hline & & $\begin{array}{l}\text { and retrieval } \\
\text { using } \\
\text { methods } \\
\text { such as } \\
\text { Named } \\
\text { Entity } \\
\text { Recognition, } \\
\text { Co-reference } \\
\text { Resolution, } \\
\text { etc. }\end{array}$ & $\begin{array}{l}\text { machine- } \\
\text { processed. }\end{array}$ \\
\hline Advantages & $\begin{array}{l}\text { 1. Provides a } \\
\text { very high } \\
\text { level of } \\
\text { accuracy. }\end{array}$ & $\begin{array}{l}\text { 1. Provides a } \\
\text { reasonably } \\
\text { high level of } \\
\text { accuracy. } \\
\text { 2. Also } \\
\text { provides a } \\
\text { means for } \\
\text { humans to } \\
\text { make } \\
\text { corrections } \\
\text { where } \\
\text { necessary. }\end{array}$ & $\begin{array}{l}\text { 1. Quick. } \\
\text { 2. Feasible } \\
\text { as a solution } \\
\text { for web data } \\
\text { annotation. } \\
\text { 3. A self- } \\
\text { learning } \\
\text { iterative } \\
\text { annotation } \\
\text { process } \\
\text { would } \\
\text { provide a } \\
\text { very high } \\
\text { level of } \\
\text { accuracy. }\end{array}$ \\
\hline Disadvantages & $\begin{array}{l}\text { 1. Tedious } \\
\text { and } \\
\text { cumbersome } \\
2 \text {. Time- } \\
\text { Consuming } \\
\text { 3. Long- } \\
\text { Winded } \\
4 \text {. Not } \\
\text { feasible for } \\
\text { the } \\
\text { annotation } \\
\text { of over } 7 \\
\text { billion web } \\
\text { pages }\end{array}$ & $\begin{array}{l}\text { 1. Quite } \\
\text { tedious and } \\
\text { time- } \\
\text { consuming } \\
2 . \text { Not ideal } \\
\text { for } \\
\text { annotating } \\
\text { the vast } \\
\text { amount of } \\
\text { web data. }\end{array}$ & $\begin{array}{l}1 . \\
\text { Processing- } \\
\text { Overload: It } \\
\text { requires a lot } \\
\text { of machine } \\
\text { processing } \\
\text { capabilities } \\
\text { and } \\
\text { dynamism } \\
\text { for machine } \\
\text { resources } \\
\text { allocation } \\
\text { for on- } \\
\text { demand } \\
\text { semantic } \\
\text { annotation. }\end{array}$ \\
\hline
\end{tabular}

\section{SEMANTIC ANNOTATION TOOLS}

There are numerous semantic annotation tools that have been developed over the years. While some have become obsolete and unavailable, several others are still available for use albeit with challenges. These tools vary in the development and usage mode across the diverse types of techniques for implementing semantic annotation. Despite the available number of tools, semantic annotation is still a daunting task and not readily available for applications on the web. This is due to the limitations present in the tools. The presence of these limitations is quite understandable as there has been too little effort into the evolvement of semantic annotation in line with technological innovations in the industry. Very little has been done to leverage recent and trendy technological 
innovations, such as cloud computing, autonomic computing, Internet of Things, etc. for semantic annotation and since the tools were not built with any of these technologies in mind, the tools have struggled in recent years to deliver. To the best of the authors' knowledge, the most comprehensive reviews of semantic annotation tools based on a set of requirements were reported by [4].

From the review presented by [4], it can be observed that the existing semantic annotation tools do not meet a substantial amount of the requirements and hence, do not possess the capabilities to deliver semantic annotation on a large-scale basis. From the review, only SHOE Knowledge Annotator, SMORE, COHSE Annotator and MnM make use of an ontology server to provide ontology support. An ontology server has the capability to accept third-party ontologies for annotation data generation as it is very vital for a semantic annotation tool [4]. Out of these four tools, only one (COHSE Annotator) provides annotation data over a server and while it does, its standard format (DAMIL + OIL) provides a very low level of expressivity compared to RDFS and OWL. The other tools embed annotation within web pages which implies that the annotation data cannot be shared and re-used by web pages offering similar content. There are also issues with usercentered designs and document evolution for most of these tools. Another review by [20] was based on a slightly separate set of requirements but further reveals the limitations in the existing tools. From the latter review, while most of the tools are dynamic in nature, there are still annotation storage and scalability issues prominent across them.

From the reviews referred to above regarding the most popular semantic annotation tools currently and their processes, it can be observed that none of them meets the requirements for a semantic annotation on-the-fly tool which can be generally deployed and utilised on a large scale for the Web. Features such as dynamicity for ontologies and annotation data are generally missing in these tools. Also, the tools only focus on the semantic annotation process, with little or no features for ontology engineering activities (such as ontology mapping) to provide rich-content annotation data. While some (such as Seeker) have adopted the decoupled annotation data approach to foster large scale semantic annotation, the data is still static and consistency between ontologies and web documents unavailable.

Another major issue observed is the close integration of most of the tools (such as KIM) with a specific knowledgebase or ontology, thereby making them unusable by other third-party ontologies [21]. Additional services such as annotation data sharing, re-usage and co-location are also missing from the existing tools. Based on these, some limitations in existing tools are as follows:

- Most tools still implement a manual (or semiautomatic) method of semantic annotation, thereby making the process very tedious and cumbersome; without the possibility of a large-scale use.
- For most automatic tools, the automation technique (or algorithm) can be improved upon by the implementation of a hybrid technique; utilizing both machine-learning and rule-based techniques for better results.

- Most of the tools implement semantic annotation using a specific knowledgebase or ontology. This is a limitation as it limits the scope of the resources that can utilize the tool to those within the same domain as the knowledgebase (or ontology). In such cases, the annotation data (or metadata) can only be as good as the knowledgebase. Furthermore, while some other tools utilize more than a single ontology, there are only restricted to a set of ontologies specific to the tool. The capability to integrate third-party ontologies is very vital to a semantic annotation tool.

- Rich-content annotation data is not always available as most tools do not implement ontology engineering techniques such as ontology mapping, merging, alignment, etc. to make the best use of several ontologies within a specific domain.

- Annotation Data Storage is better stored on an annotation server for sharing and re-use by several similar web applications. For most of the tools, it is local to the web resource it has been generated for in most cases, thereby implying other similar web resources cannot utilize it as well.

- None of the tools provide an automated means of the provision of annotation data for web applications onthe-fly.

\section{THE CASE FOR A CLOUD-DRIVEN SEMANTIC WEB}

The online, real-time automation of semantic annotation for billions of web pages dynamically would require a vast amount of computing power and a high level of automation [14]. While data is generally being migrated to cloud platforms to leverage cloud computing capabilities, the cloud is envisaged as being able to provide a technical solution to the computing resources' overload challenge. One of the reasons is because since the semantic web is about automation of processes, it makes it an option to implement it on a cloud environment as the cloud computing paradigm on which cloud environments are built is also based on services automation.

Furthermore, with the availability of vast amount of scalable resources on cloud, such as CPU, RAM and other physical storages, metadata processing for the semantic web can become enhanced, scalable and distributed. It is evident that that cloud computing provides several advantages over other traditional methods of computing. These advantages can be transferred to the semantic web when it is driven by a cloud facility. These advantages include reliability, scalability, highlevel of security, easier access and retrieval of data, handling 
and processing of vast amounts of data, load balancing among applications, etc. [22].

With the capabilities of the PaaS (Platform-as-a-Service) model of cloud computing and the availability of virtualization, a cloud-driven platform can be implemented to provide real-time, on-demand, self-service semantic annotation to web documents, which has remained a daunting task with traditional computing paradigms. With increase in data migration to the cloud as well as web hosting companies offering cloud-based web hosting, it has become a general view among professionals both in the industry and academia that the web will eventually be hosted in the cloud; making it possible for web-based applications to benefit from the many advantages of cloud environments. This is demonstrated with the diagram below:

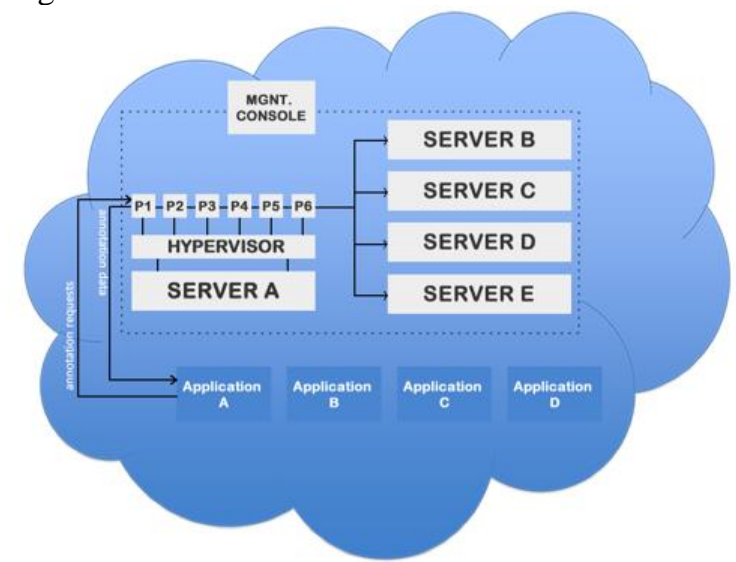

Figure 4 - Virtualization in the Cloud for Semantic Annotation

With the use of virtualization technology, instances of virtual machines can be set up to meet the high demands of online automatic semantic annotation. Likewise, the annotation can be delivered as a service with a service model mechanism that makes use of both specialized and generic cloud mechanisms such as automated scaling listener, live VM migration, cloud workload scheduler, virtual infrastructure manager, billing management system, etc. to provide the service [14]. Furthermore, since the semantic web is about automation of processes, it makes it an option to implement it on a cloud environment as they are built based on automation of services.

Also, the use of cloud computing mechanisms is of importance in the realization of the semantic web. These mechanisms allow for the provision of IT solutions such as some of those faced by the semantic web. Such mechanisms include the cloud consumer gateway, cloud storage data placement auditor, cloud storage data aging management, cloud storage performance, cloud usage monitor, cloud storage management portal, cloud workload scheduler, and various cloud storage devices. Hence, revisiting the list of some limitations in existing semantic annotation tools (in section 5), the table below provides a description of how the cloud can be leveraged for some of the limitations:

Table 2 - Leveraging Cloud for Semantic Annotation Challenges

\begin{tabular}{|l|}
\hline Limitation \\
\hline Most tools still implement a \\
manual (or semi-automatic) \\
method of semantic \\
annotation, thereby making \\
the process very tedious and \\
cumbersome; without the \\
possibility of a large-scale \\
use.
\end{tabular}

Most of the tools implement semantic annotation using a specific knowledgebase or ontology. This is a limitation as it limits the scope of the resources that can utilize the tool to those within the same domain as the knowledgebase (or ontology). In such cases, the annotation data (or metadata) can only be as good as the knowledgebase. The capability to integrate third-party ontologies is very vital to a semantic annotation tool.

Rich-content annotation data is not always available as most tools do not implement ontology engineering techniques such as ontology mapping, merging, alignment, etc. to make the best use of several ontologies within a specific domain.

Annotation Data Storage is better stored on an annotation server for sharing and re-use by several similar web applications. For most of the tools, it is local to the web resource it has been generated for in most cases, thereby implying other similar web resources cannot utilize it as well.

None of the tools provide an automated means of the provision of annotation data for web applications on-thefly.

A cloud-service-based
subscription for annotation
data based on cloud's pay-as-
you-go capability would
enable online, real-time
annotation of web documents
on the fly.

\section{CONCLUSION}

This paper has focused on how cloud computing as a technology can provide leverage for large-scale semantic annotation, thereby facilitating the emergence of a truly 
semantic Web 3.0. From the analysis, it is believed that cloud computing can facilitate semantic annotation and should be extensively utilised for this purpose. A cloud-driven semantic annotation platform can provide a basis for the delivery of semantic annotation as a cloud service, with web applications subscribing to its usage. The required extensive data processing (for metadata) and its annotation to web applications as-aservice and on a subscription basis are features that a cloud platform can facilitate and deliver on a large scale. However, further research is required to identify, analyse and provide a matrix of how specific cloud entities will facilitate each of the processes required for implementing semantic annotation. Finally, it is also pertinent to extensively research into how annotation data (i.e. metadata) can be further enriched and optimised because the availability of a cloud-driven platform for semantic annotation would need to be complemented with rich-content metadata to obtain the best results of effectiveness and efficiency.

\section{REFERENCES}

[1] Gunelius, S., 2014. The Data Explosion in 2014 Minute by Minute. 12 July 2014. Available through the world wide web: http://aci. info/2014/07/12/the-data-explosion-in-2014-minute-by-minuteinfographic.

[2] Siva, M. and Poobalan, A., 2012. Semantic Web Standard in Cloud Computing. International Journal of Soft Computing and Engineering, 1, pp.1-5.

[3] Fensel, D., Morozova, O., 2010. Resource Description Framework. Semantic Technology Institute, University of Innsbruck, Austria.

[4] Uren, V., Cimiano, P., Iria, J., Handschuh, S., Vargas-Vera, M., Motta, E. and Ciravegna, F., 2006. Semantic annotation for knowledge management: Requirements and a survey of the state of the art. Web Semantics: science, services and agents on the World Wide Web, 4(1), pp.14-28.

[5] Wenyu, Z. \& Yin, J., 2008. Exploring Semantic Web Technologies for ontology-based modelling in collaborative engineering design. IEEE Industrial Electronics Magazine. Vol 36. (Jan.). p. 833 - 843

[6] Hogan, A., Harth, A., Umbrich, J., Kinsella, S., Polleres, A. and Decker, S., 2011. Searching and browsing linked data with SWSE: The semantic web search engine. Web semantics: science, services and agents on the world wide web, 9(4), pp.365-401.

[7] Yates, A., 2007. Information extraction from the web: Techniques and applications (Vol. 68, No. 11).

[8] Dill, S., Eiron, N., Gibson, D., Gruhl, D., Guha, R., Jhingran, A., Kanungo, T., Rajagopalan, S., Tomkins, A., Tomlin, J.A. and Zien, J.Y.,
2003, May. SemTag and Seeker: Bootstrapping the semantic web via automated semantic annotation. In Proceedings of the 12th international conference on World Wide Web (pp. 178-186). ACM.

[9] Tjoa, A.M., Andjomshoaa, A., Shayeganfar, F. and Wagner, R., 2005, August. Semantic Web challenges and new requirements. In Database and Expert Systems Applications, 2005. Proceedings. Sixteenth International Workshop on (pp. 1160-1163). IEEE.

[10] Calvanese, D., De Giacomo, G. and Lenzerini, M., 2001, July. A framework for ontology integration. In Proceedings of the First International Conference on Semantic Web Working (pp. 303-316). CEUR-WS. org.

[11] Benjamins, R., Contreras, J., Corcho, O. and Gomez-Perez, A., 2002. The six challenges of the Semantic Web.

[12] Burstein, M.H. and McDermott, D.V., 2005. Ontology translation for interoperability among semantic web services. AI magazine, 26(1), p.71.

[13] Guarino, N., Oberle, D., Staab, S., 2011. What is an Ontology? [Online] Available from http://iaoa.org/isc2012/docs/Guarino2009_What_is_an_Ontology.pdf [Accessed: 24/07/2016]

[14] Hsu, I. and Cheng, F.Q., 2015. SAaaS: a cloud computing service model using semantic-based agent. Expert Systems, 32(1), pp.77-93.

[15] Fensel, D., 2010. Generating Semantic Annotations. Semantic Technology Institute, University of Innsbruck, Austria.

[16] Dammak, S.M., Jedidi, A. and Bouaziz, R., 2013, December. Automation and evaluation of the semantic annotation of Web resources. In Internet Technology and Secured Transactions (ICITST), 2013 8th International Conference for (pp. 443-448). IEEE.

[17] Jellouli, I. and El Mohajir, M., 2009, April. Towards automatic semantic annotation of data rich Web pages. In Research Challenges in Information Science, 2009. RCIS 2009. Third International Conference on (pp. 139-142). IEEE.

[18] Leida, M. and Chu, A., 2013, June. Distributed SPARQL query answering over RDF data streams. In Big Data (BigData Congress), 2013 IEEE International Congress on (pp. 369-378). IEEE.

[19] Kiryakov, A., Popov, B., Terziev, I., Manov, D. and Ognyanoff, D., 2004. Semantic annotation, indexing, and retrieval. Web Semantics: Science, Services and Agents on the World Wide Web, 2(1), pp.49-79.

[20] Rodríguez-García, M.Á., Valencia-García, R., García-Sánchez, F. and Samper-Zapater, J.J., 2014. Creating a semantically-enhanced cloud services environment through ontology evolution. Future Generation Computer Systems, 32, pp.295-306.

[21] Reeve, L. and Han, H., 2005, March. Survey of semantic annotation platforms. In Proceedings of the 2005 ACM symposium on Applied computing (pp. 1634-1638). ACM.

[22] Vázquez-Poletti, J. L., Moreno-Vozmediano, R., Han, R., Wang, W., \& Llorente, I. M., 2017. SaaS enabled admission control for MCMC simulation in cloud computing infrastructures. Computer Physics Communications, 211, 88-97. 